\title{
BIFURCATION PHENOMENA FOR AN OXIDATION REACTION IN A CONTINUOUSLY STIRRED TANK REACTOR. III THE INHIBITING EFFECT OF AN INERT SPECIES
}

\author{
M. I. NELSON ${ }^{1}$
}

(Received 1 June, 2003; revised 25 March, 2004)

\begin{abstract}
We extend an investigation into the static and dynamic multiplicity exhibited by the reaction of a fuel/air mixture in a continuously stirred tank reactor by considering the effect of adding a chemically inert species to the reaction mixture. The primary bifurcation parameter is taken to be the fuel fraction as this is the most important case from the perspective of fire-retardancy. We show how the addition of the inert species progressively changes the steady-state diagrams and flammability limits. We also briefly outline how heat-sink additives can be incorporated into our scheme.
\end{abstract}

\section{Introduction}

In the first two parts of this series $[12,13]$ the static and dynamic multiplicity for a simple oxidation reaction occurring in a continuously stirred tank reactor (CSTR) was investigated. The chemical mechanism consists of a two-state decay of a precursor through a reactive intermediate to a final product. The second stage is an oxidation reaction that is first order with respect to both reactants:

$$
\begin{aligned}
\mathscr{F} \stackrel{k_{1}}{\rightarrow} \mathscr{B}, & Q_{1}=0, \\
\mathscr{B}+\mathscr{O}_{2} \stackrel{k_{2}(T)}{\longrightarrow} \mathscr{C}, & Q_{2} \neq 0 .
\end{aligned}
$$

Normally in CSTR studies, the total flow rate is often chosen as the primary bifurcation parameter. In this paper we investigate how the response of the system changes as the composition of the reaction mixture is varied at a constant flow rate. We show how knowledge of the static and dynamic bifurcation behaviour exhibited by a fuel-air

\footnotetext{
'School of Mathematics and Applied Statistics, University of Wollongong, Wollongong, NSW 2522, Australia; e-mail: nelsonm@member.ams.org.

(C) Australian Mathematical Society 2005, Serial-fee code 1446-1811/05
} 
mixture provides a framework to investigate the change in behaviour when the reaction mixture is diluted at constant pressure by the addition of a chemically inert species. The choice of mechanism used in this study is not important, since the aim of the paper is to illustrate the proposed method: the same method would be used if a more detailed chemical mechanism were used.

Gas-phase active fire retardants can be broadly classified as being either chemical or physical in their mechanism. The distinction between these classes is that a gas-phase active chemical fire-retardant 'interferes' chemically with the mechanism of flame propagation. In this paper we investigate the change in behaviour of our system as the reaction-mixture is diluted by the addition of a chemically inert species.

Although in this paper we are considering the simplest possible mode of action for an additive with a very simple chemical mechanism, our methodology can be applied to more realistic oxidation mechanisms and more detailed additive chemistry.

1.1. Summary of previous work In this section we summarise those aspects of our earlier work $[12,13]$ that are pertinent to the current investigation. This corresponds to the choice of the fuel fraction $(\alpha)$ as the primary bifurcation parameter, with the inflow temperature $\left(T_{0}\right)$ and inflow pressure $(\mathscr{P})$ as the secondary bifurcation parameters. The fuel fraction is the ratio of the pressure (concentration) of the pre-cursor species flowing into the reactor to the total pressure flowing into the reactor:

$$
\alpha=\frac{\mathscr{P}_{F}}{\mathscr{P}_{\mathscr{F}}+\mathscr{P}_{\mathrm{i}}+\mathscr{P}_{\text {air }}} .
$$

In general the model for reactions (1.1) and (1.2) consists of a temperature equation and an equation each for the three chemical species $\mathscr{F}, \mathscr{B}$ and $\mathscr{O}_{2}$.

In [12] Nelson and Sidhu investigated the behaviour of the oxidation mechanism (1.1) and (1.2) in an adiabatic reactor. In this case the reactor model can be reduced to a single ordinary differential equation (ODE). Periodic solutions are therefore impossible in an adiabatic reactor. The organising centre for the static bifurcation problem is a pitchfork singularity, consequently there are four generic steady-state diagrams. One of these is the isola, an example of which is shown in Figure 1. This contains two disjoint solution branches: a low-valued unique no-ignition branch and an isola, which has stable and unstable branches. The isola has two extinction limit points at which combustion is extinguished; between these points the flame temperature varies smoothly with the fuel fraction. Flammability limits are identified with the extinction limit points.

In [13], Nelson and Sidhu investigated the behaviour of the oxidation mechanism (1.1) and (1.2) in a diabatic reactor. In this case the reactor model can be reduced to a system of two coupled ODEs. The pitchfork singularity remains the organising centre for the static bifurcation problem. However, Hopf bifurcations now occur. Over the 


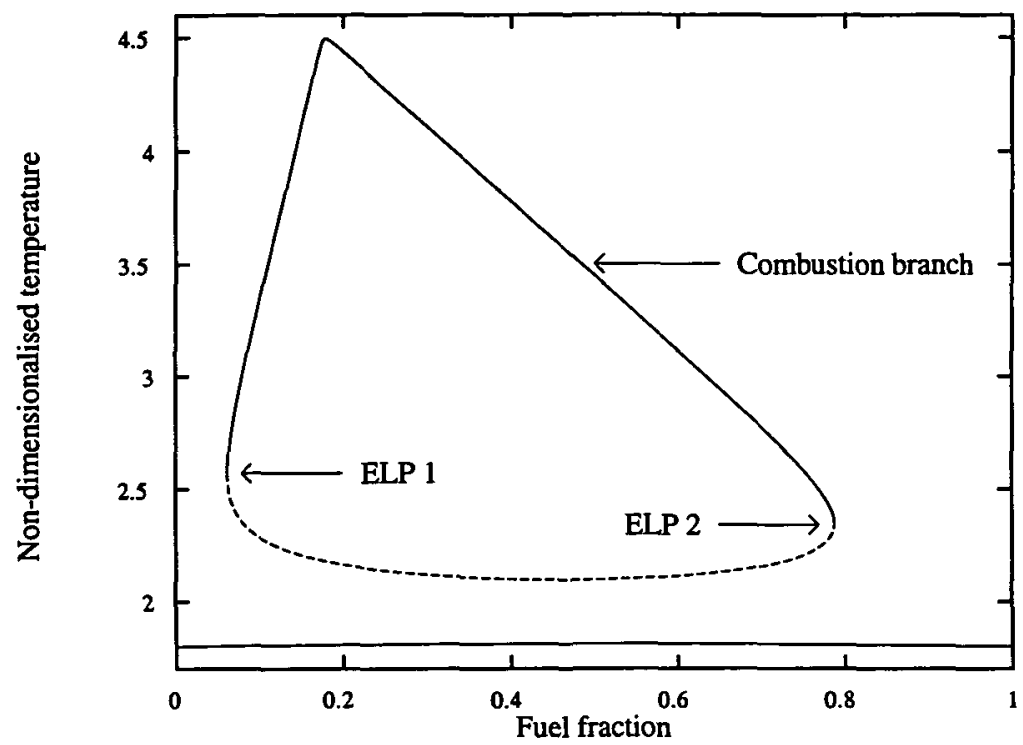

FIGURE 1. A typical isola steady-state diagram for the adiabatic reactor. Parameter values: dimensionless precursor decay rate, $A_{1}^{*}=0.1$; dimensionless pressure, $\mathscr{P}^{*}=0.02$; dimensionless inflow temperature, $T_{0}^{*}=1.8$; heat-transfer parameter, $J=0$. Nomenclature: ELP=Extinction limit point. Figure reprinted from [12], $C$ Kluwer Academic/Plenum Publishing Corporation.

parameter range considered, eight generic steady-state diagrams based on the isola structure were found. Half of these do not describe flammability in the accepted sense as the 'no-ignition' unique branch loses/gains stability at one of two Hopf points. Of the remaining steady-state diagrams one does not represent flammability as both branches on the isola are unstable. The three 'flammable' steady-state diagrams differ from the flammability scenario represented by Figure 1 in that only part of the ignition branch is stable. Stability is no longer gained/lost at an extinction limit point but at a supercritical Hopf bifurcation. Accordingly there is a parameter region in which oscillations of near-extinction flames are observable.

\section{Model equations}

We consider gaseous chemical species $\mathscr{F}$ and $\theta_{2}$ flowing separately into a continuously stirred tank reactor subject to the chemical mechanism (1.1) and (1.2). The CSTR is assumed to be contained within a cooling system in which the coolant flow rate can be quickly manipulated so as to obtain tight control of the coolant temperature. This means that a differential equation for the coolant temperature is not required and it can be regarded as a free parameter. Heat transfer between the cooled reactor walls 
and the interior of the reactor is modelled by Newtonian cooling. We use the standard hypotheses that the reactor vessel has constant volume, perfect mixing and constant physical properties. The relationship between the model and experimental procedures is described in [12].

The assumption that the reactants enter into the reactor separately through two inlets is not crucial. By rescaling variables, the governing equations can be transformed to model an experiment in which the reactants enter the reactor through a single inlet [14].

The dimensional variables are scaled using a reference temperature $\left(T^{*}=T / 298\right)$, a reference concentration (the concentration of an ideal gas at atmospheric pressure and a temperature of $298 \mathrm{~K}$ ) and a timescale based upon Newtonian cooling. Full details are provided elsewhere [12]. The system that we study is

Dimensionless precursor concentration

$$
\frac{\mathrm{d} \mathscr{F}^{*}}{\mathrm{~d} t^{*}}=q_{1}^{*} \alpha-\left(q_{1}^{*}+q_{2}^{*}\right) \mathscr{F}^{*}-A_{1}^{*} \mathscr{F}^{*},
$$

Dimensionless fuel concentration

$$
\frac{\mathrm{d} \mathscr{B}^{*}}{\mathrm{~d} t^{*}}=-\left(q_{1}^{*}+q_{2}^{*}\right) \mathscr{B}^{*}+A_{1}^{*} \mathscr{F}^{*}-\frac{A_{2}^{*} \mathscr{P}^{*}}{T_{0}^{*}} e^{-E_{2}^{*} / \tau^{*}} \mathscr{B}^{*} \mathscr{O}_{2}^{*},
$$

Dimensionless oxygen concentration

$$
\frac{\mathrm{d} \mathscr{O}_{2}^{*}}{\mathrm{~d} t^{*}}=0.21 q_{2}^{*}(1-\alpha-\gamma)-\left(q_{1}^{*}+q_{2}^{*}\right) \mathscr{O}_{2}^{*}-\frac{A_{2}^{*} \mathscr{P}^{*}}{T_{0}^{*}} e^{-E_{2}^{*} / T^{*}} \mathscr{B}^{*} \mathscr{O}_{2}^{*},
$$

Dimensionless inert concentration

$$
\frac{\mathrm{d} \mathscr{I}^{*}}{\mathrm{~d} t^{*}}=q_{2}^{*} \gamma-\left(q_{1}^{*}+q_{2}^{*}\right) \mathscr{I}^{*}
$$

Dimensionless temperature

$$
\frac{\mathrm{d} T^{*}}{d t^{*}}=\left(q_{1}^{*}+q_{2}^{*}\right)\left(T_{0}^{*}-T^{*}\right)+\frac{Q_{2}^{*} A_{2}^{*} \mathscr{P}^{*^{2}}}{T_{0}^{*^{2}}} e^{-E_{2}^{*} / T^{*}} \mathscr{B}^{*} \mathscr{O}_{2}^{*}-J\left(T^{*}-T_{a}^{*}\right),
$$

and Dimensionless initial conditions

$$
\begin{aligned}
\mathscr{F}^{*}(0) & =\mathscr{B}^{*}(0)=\mathscr{O}_{2}^{*}(0)=0, \\
T^{*}(0) & =\frac{\left(q_{1}^{*}+q_{2}^{*}\right) T_{0}^{*}+J T_{a}^{*}}{q_{1}^{*}+q_{2}^{*}+J} .
\end{aligned}
$$

The terms appearing in (2.1)-(2.7) are defined in the Nomenclature. In (2.3) the number 0.21 is the fraction of oxygen in the air flowing through the reactor [12].

We assume that prior to the commencement of the experiment the entire system is first flushed with an inert species at temperature $T_{0}^{*}$. Thus the initial concentration of the chemical species in (2.6) is zero whilst the initial temperature of the reactor in (2.7) is the corresponding steady-state solution. 
There is a one-to-one relationship between our dimensionless variables and their dimensional counterparts. Hence we often write 'inflow pressure' rather than 'dimensionless inflow pressure'. The experimentally controllable parameters are the reactant composition $(\alpha)$ and $(\gamma)$, the inflow pressure $\left(\mathscr{P}^{*}\right)$, the inflow temperature $\left(T_{0}^{*}\right)$, the inflow rates $\left(q_{1}^{*}\right.$ and $\left.q_{2}^{*}\right)$ and the vessel wall temperature $\left(T_{a}^{*}\right)$. The non-controllable parameters are: the rate constant for the decay of the precursor $\left(A_{1}^{*}\right)$, the pre-exponential factor, activation energy and exothermicity for the oxidation reaction $\left(A_{2}^{*}, E_{2}^{*}\right.$ and $Q_{2}^{*}$ respectively) and a dimensionless constant controlling the amount of heat-transfer in the system $(J)$.

In what follows we assume that the inflow temperature $\left(T_{0}^{*}\right)$ is equal to the temperature of the reactor walls $\left(T_{a}^{*}\right)$. We also assume that the inflow rates are equal $\left(q_{1}^{*}=q_{2}^{*}\right)$. We take the fuel fraction $(\alpha)$ as the primary bifurcation parameter. The possible secondary bifurcation parameters are the inert fraction $(\gamma)$, the inflow pressure $\left(\mathscr{P}^{*}\right)$ and the inflow temperature $\left(T_{0}^{*}\right)$.

Note that (2.1) and (2.4) may be integrated to give the concentrations of the precursor and inert species respectively as a function of time. Thus the set of five model equations (2.1)-(2.5) immediately becomes a set of three equations. The fuel-air system considered in $[12,13]$ is obtained by taking $\gamma=0$ in (2.3) and (2.4). The adiabatic model considered in [12] corresponds to taking $J=0$ in (2.5).

Note from (2.1) that if $A_{1}^{*} \gg q_{1}^{*}+q_{2}^{*}$ then the steady-state concentration of the precursor species is

$$
F^{*} \approx q_{1}^{*} \alpha / A_{1}^{*}
$$

The source term $A_{1}^{* F^{*}}$ in (2.2) then has value

$$
A_{1}^{*} \mathscr{F}^{*} \approx q_{1}^{*} \alpha .
$$

The physical interpretation of this is that under these conditions the behaviour of the model system defined by reactions (1.1) and (1.2) is the same as a model in which only the oxidation reaction (1.2) occurs, the inflow concentration of fuel into the reactor being $\alpha$.

2.1. Numerics The path-following software program Auto 97 [4] was used to obtain steady-state and unfolding diagrams. In steady-state diagrams the standard representation is used: solid lines are stable steady states; dashed lines are unstable steady states; squares are Hopf bifurcation points; filled and unfilled circles are stable and unstable periodic solutions respectively.

Continuation methods require a known solution to begin calculations. Equations (2.1)-(2.5) have two natural starting places for continuation: no precursor species in the inflow $(\alpha=0)$ and no air in the inflow $(\alpha+\gamma=1)$. The appropriate steady-state 
solutions are:

$$
\begin{array}{ll}
\text { No fuel in the inflow }(\alpha=0) & \text { No air in the inflow }(\alpha+\gamma=1) \\
\mathscr{F}^{*}=0, & \mathscr{F}^{*}=\frac{q_{1}^{*}}{q_{1}^{*}+q_{2}^{*}+A_{1}^{*}} \\
\mathscr{B}^{*}=0, & \mathscr{B}^{*}=\frac{n A_{1}^{*}}{q_{1}^{*}+q_{2}^{*}} \cdot \frac{(1-\gamma) q_{1}^{*}}{q_{1}^{*}+q_{2}^{*}+A_{1}^{*}} \\
\mathscr{O}_{2}^{*}=\frac{0.21 q_{2}^{*}(1-\gamma)}{q_{1}^{*}+q_{2}^{*}}, & \mathscr{O}_{2}^{*}=0, \\
T^{*}=\frac{\left(q_{1}^{*}+q_{2}^{*}\right) T_{0}^{*}+J T_{a}^{*}}{q_{1}^{*}+q_{2}^{*}+J}, & T^{*}=\frac{\left(q_{1}^{*}+q_{2}^{*}\right) T_{0}^{*}+J T_{a}^{*}}{q_{1}^{*}+q_{2}^{*}+J} .
\end{array}
$$

\section{Results}

In Subsections 3.1 and 3.2 we consider the role of a diluent in adiabatic and diabatic reactors respectively.

\subsection{Adiabatic reactor $(J=0)$}

3.1.1. Reduction of the model to a single $O D E$ By applying the procedure used in [12] it is possible to reduce (2.2), (2.3) and (2.5) to a single ordinary differential equation. Oscillatory behaviour is therefore impossible for a fuel/air/inert mixture under adiabatic conditions. This reduction is possible because under adiabatic conditions the model has two invariant relationships.

The existence of these relationships may be explained as follows. As (2.1) can be solved, we can calculate the concentration of the fuel species within the reactor in the absence of oxidation. Suppose that we measure the concentration of fuel in the reactor. We now know how much fuel has been consumed through oxidation. As the concentration of oxygen entering the reactor is known, the oxygen concentration can be calculated from the measured fuel concentration. Thus if we measure the fuel concentration inside the reactor we do not need to measure the oxygen concentration. This is the first invariant relationship. The second invariant relationship is a consequence of the lack of heat loss in the adiabatic model: all the energy released by the oxidation reaction is used to increase the temperature of the reaction mixture. The temperature increase is the quotient of the energy released to the heat capacity. The energy released in the oxidation reaction is determined by how much fuel has been consumed. This is a known quantity and provides the second invariant relationship. Thus if we measure the fuel concentration inside the reactor we do not need to measure the reactor temperature. 


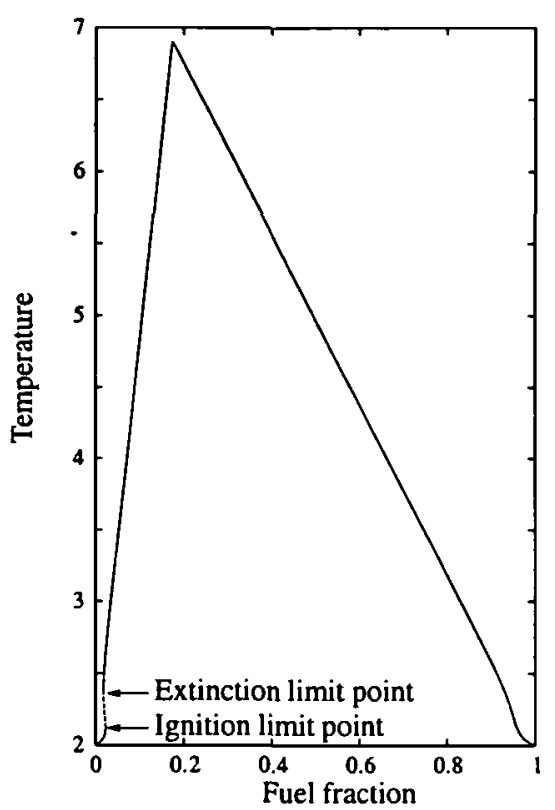

(a) Steady-state diagram in the absence of an additive $(\gamma=0)$.

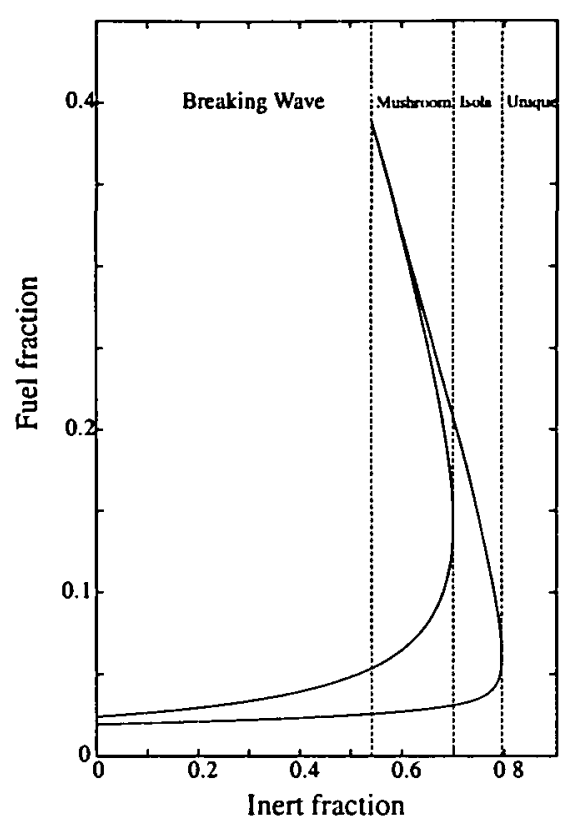

(b) Unfolding diagram as additive concentration is increased.

FIGURE 2. The change in the steady-state structure as the reaction mixture is diluted with an inert species. Parameter values: dimensionless inflow pressure $\mathscr{P}^{*}=0.04$; dimensionless inflow temperature $\mathscr{T}_{0}^{*}=2$.

The static multiplicity of the adiabatic model is governed by the steady-state solution of a single ordinary differential equation. The singularity function for the adiabatic model is given by

$$
\begin{aligned}
\mathscr{G}= & \left(q_{1}^{*}+q_{2}^{*}\right)\left(T_{0}^{*}-T^{*}\right) \\
& +\frac{Q_{2}^{*} A_{2}^{*} \mathscr{P}^{*^{2}}}{T_{0}^{*^{2}}} e^{-E_{2}^{*} / T^{*}}\left\{\frac{n q_{1}^{*} \alpha A_{1}^{*}}{\left(q_{1}^{*}+q_{2}^{*}\right)\left(q_{1}^{*}+q_{2}^{*}+A_{1}^{*}\right)}+\frac{T_{0}^{*}}{Q_{2}^{*} \mathscr{P P}^{*}}\left(T_{0}^{*}-T^{*}\right)\right\} \\
& \times\left\{\frac{0.21 q_{2}^{*}(1-\alpha-\gamma)}{q_{1}^{*}+q_{2}^{*}}+\frac{T_{0}^{*}}{Q_{2}^{*} \mathscr{P P}^{*}}\left[T_{0}^{*}-T^{*}\right]\right\} .
\end{aligned}
$$

When $\gamma=0$ this equation reduces to [12, Equation (38)].

3.1.2. Analysis of the model In this section we show how the steady-state diagram of the system changes as the inert fraction $(\gamma)$ is increased. Figure 2 (a) shows a steady-state diagram for a fuel/air mixture in the absence of the inert component 


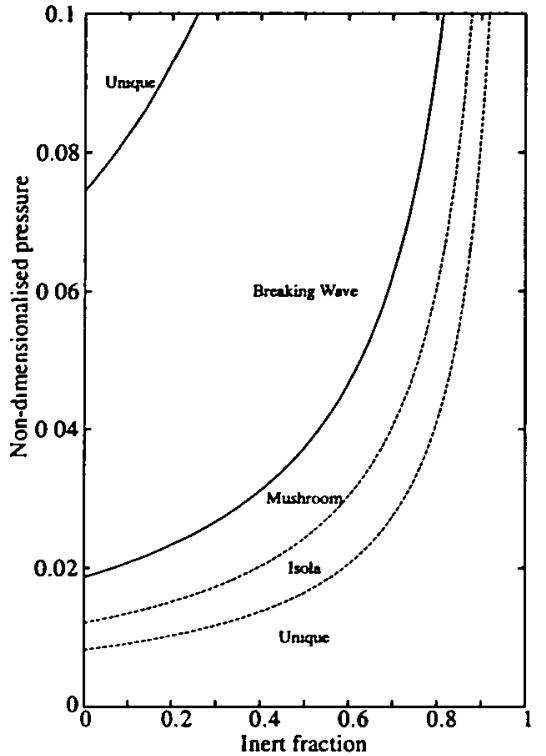

(a) Bifurcation diagram for fuel-airinert mixtures at fixed inflow temperature.

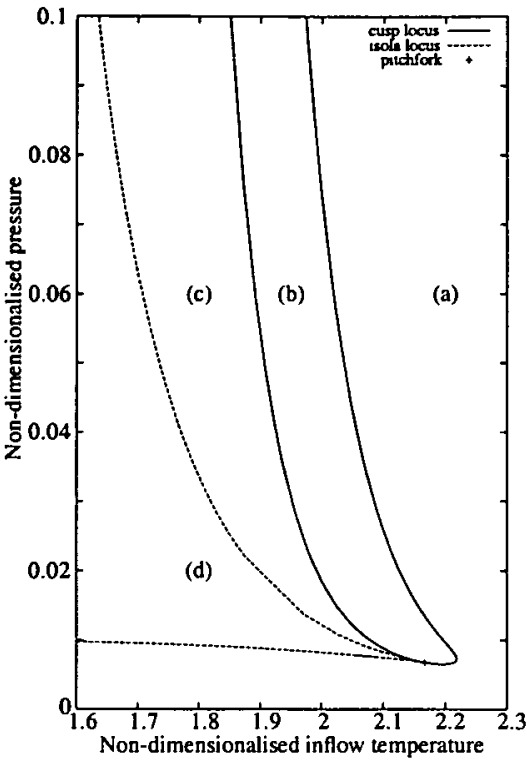

(b) Bifurcation diagram for fuel-air mixtures when $\gamma=0$.

FIGURE 3. Bifurcation diagrams in an adiabatic reactor. In figure (b) the marked regions correspond to: (a) a unique steady-state; (b) a single hysteresis loop (breaking wave); (c) a double hysteresis loop (mushroom); and (d) an isola. Figure 2 (b) is a cross-section through figure (a) at an inflow pressure $\mathscr{P}^{*}=0.04$. Parameter values: (a) dimensionless inflow temperature $\mathscr{T}_{0}^{*}=2$. Figure (b) reprinted from [12], (CKluwer Academic/Plenum Publishing Corporation.

$(\gamma=0)$. This diagram is of the 'breaking-wave' type, containing one ignition limit point and one extinction limit point. From a practical perspective the main question of interest is how the values of the limit points change as the inert fraction increases. This question is answered by unfolding the ignition limit point with the inert fraction. The results of such an unfolding are shown in Figure 2 (b). This shows that as the fuel fraction is increased the steady-state structure passes through the sequence breaking waves to mushroom to isola to unique. For sufficiently large values of the inert fraction $\left(\gamma_{c r} \approx 0.8\right)$ the fuel-air components of the resulting fuel/air/inert mixtures are sufficiently diluted that the heat release rate is insufficient to support sustained combustion: the reactions mixture is not combustible.

Figure 3 (a) is a bifurcation diagram for fuel-air-inert systems at a fixed inflow temperature. Note that the small unique region in the top left-hand corner of this figure represents a combustion hazard even though the steady-state diagram contains no limit 
points due to the high temperatures that are generated by the reaction. For such systems criticality can be defined using sensitivity analysis [12]. The 'lower' unique region represents a region of safety. Figure 3 (a) shows that as the inflow pressure increases, the critical value of the inert fraction at which the isola-unique transition occurs rapidly increases. For a given pressure, the value of the inert fraction at which the transition between generic steady-state diagrams occurs in this figure can be obtained from the bifurcation diagram when $\gamma=0$, which is Figure 3 (b). This follows from observing that increasing the inert fraction at fixed pressure (concentration) decreases the total pressure (concentration) of fuel/air. Consequently increasing $\gamma$ in Figure 3 (a) is equivalent to decreasing the total pressure in Figure 3 (b).

Consider a point in region (b) of Figure $3(\mathrm{~b})$ with coordinates $\left(T_{0, s}^{*}, \mathscr{P P}_{s}^{*}\right)$. This point represents a system having a breaking-wave steady-state structure. Recall that the inert fraction in this system is zero $(\gamma=0)$. Now suppose that an inert species is added to the mixture whilst fuel and air are simultaneously removed so that the pressure remains constant. At what value of the inert fraction does the steady-state diagram change to an isola? To answer this question draw a vertical line from the point $\left(\mathscr{P}_{s}^{*}, T_{0, s}^{*}\right)$ to the point $\left(0, T_{0, s}^{*}\right)$. Let $\left(\mathscr{P}_{1}^{*}\right)$ be the value of the pressure at which the isola region is entered along this line, that is, the transition point between regions (c) and (d) on Figure 3 (b). The required value of the inert fraction is

$$
\gamma=1-\mathscr{P}_{i}^{*} / \mathscr{P}_{s}
$$

Thus the critical values of the inert fraction that is required to induce a transition in the bifurcation diagram for the fuel-air-inert system are determined purely by the behaviour of the fuel-air system.

\subsection{Diabatic reactor $(J=1)$}

3.2.1. Reduction of the model to two coupled ODEs Of the two invariant relationships that exist for the adiabatic system, only the first holds for the diabatic system. Thus it is now only possible to reduce the size of the system from three ordinary differential equations to two. Applying the procedure used in [13] the following system is obtained:

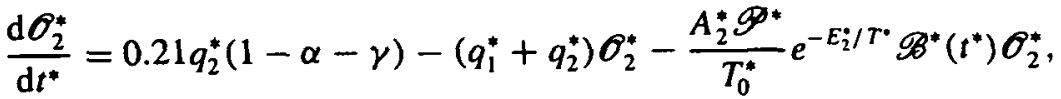

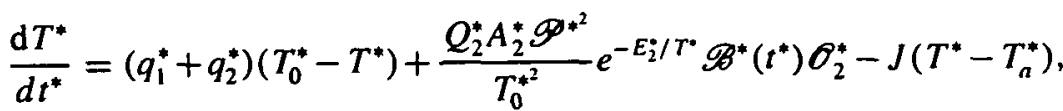

Algebraic relationship

$$
\mathscr{B}^{*}\left(t^{*}\right)=\mathscr{O}_{2}^{*}\left(t^{*}\right)+\frac{q_{1}^{*} \alpha A_{1}^{*}-0.21 q_{2}^{*}(1-\alpha-\gamma)\left(q_{1}^{*}+q_{2}^{*}+A_{1}^{*}\right)}{\left(q_{1}^{*}+q_{2}^{*}\right)\left(q_{1}^{*}+q_{2}^{*}+A_{1}^{*}\right)} .
$$

When $\gamma=0$ this model reduces to [13, Equations (24)-(26)]. 


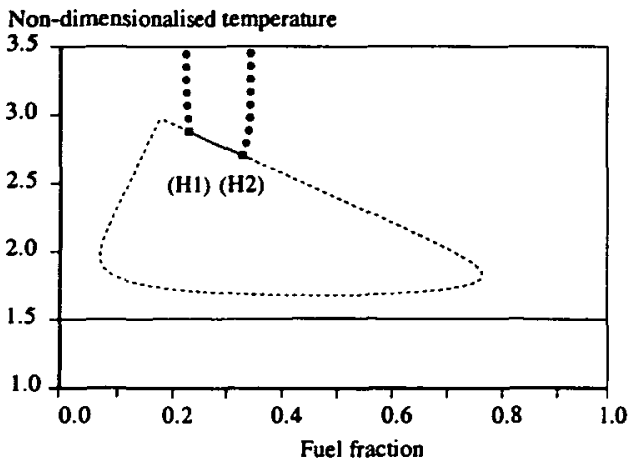

(a) Steady-state diagram.

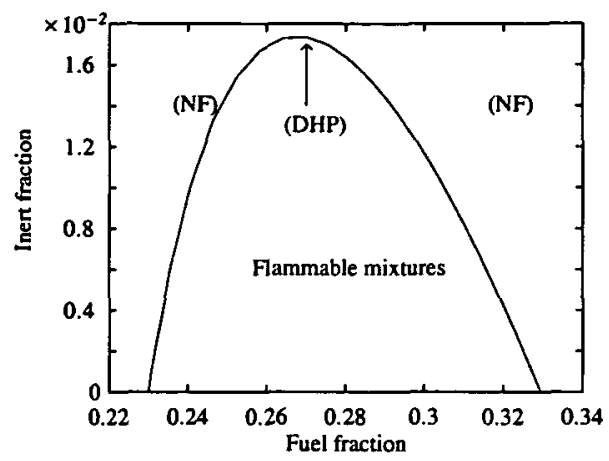

(b) Unfolding diagram.

FIGURE 4. Steady-state diagram I2a in a diabatic reactor in the absence of an inert additive $(\gamma=0)$, figure (a), and the consequent change in flammability limits as the inert fraction is increased, figure (b). Nomenclature: (NF)= Non-flammable mixture, DHP=double Hopf point. Parameter values: heat-transfer parameter, $J=1$; inflow pressure, $\mathscr{P}^{*}=4.5$; inflow temperature, $T_{0}^{*}=1.5$.

3.2.2. Analysis of the model As mentioned in Subsection 1.1, in the absence of a diluent $(\gamma=0)$ the diabatic model has three generic steady-state diagrams that represent flammability in the accepted sense. In this section we show how the addition of a diluent changes the flammability limits for two of them.

Figure 4 (a) shows the $12 a^{1}$ steady-state diagram when the inert fraction is zero. Observe that the stable flame state loses stability at supercritical Hopf bifurcations and there is a small parameter range in which stable oscillatory flames are found but no stable flame state exists. We define a mixture to be flammable if there is a stable steady-state flame. Thus the flammability limits in this figure are provided by the values of the fuel fraction at which the two Hopf bifurcation points occur.

In Figure 4 (b) the Hopf bifurcation points are unfolded with the inert fraction $(\gamma)$. As the inert fraction increases the Hopf bifurcation points approach each other. There is a critical value of the fuel fraction at which the two Hopf bifurcation points collide and destroy each other at a double Hopf point. Thus as the fuel fraction is increased through this value the fuel-air-inert mixture becomes non-flammable, that is, quenched, for any permitted value of the fuel fraction. For values of the inert fraction slightly higher than this critical value the steady state would be similar to Figure 4 (a) with the distinction that both branches of solution on the isola are entirely unstable, that is,

\footnotetext{
'The I shows that the steady-state structure is an isola. The ' 2 ' denotes that there are two Hopf bifurcation points on the isola. The ' $a$ ' shows that there are at least two steady-state diagrams that are classified as $I 2$ and this is the first. The terminology used to differentiate the steady-state diagrams is explained in [13].
} 


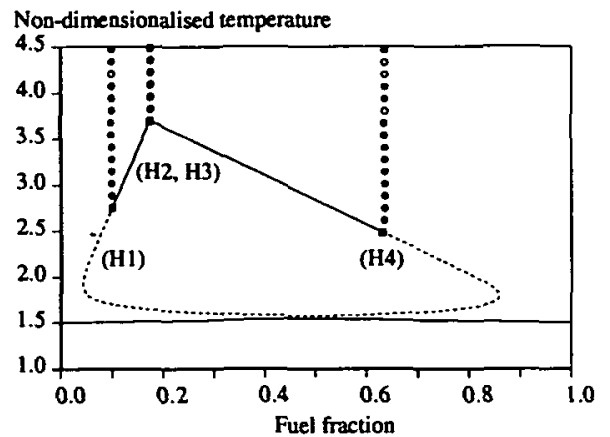

(a) Steady-state diagram.

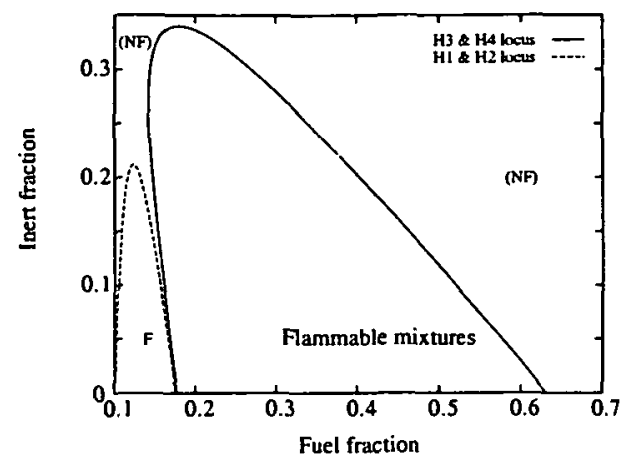

(b) Unfolding diagram.

FIGURE 5. Steady-state diagram I4 in a diabatic reactor in the absence of an inert additive $(\gamma=0)$, figure (a), and the consequent change in flammability limits as the inert fraction is increased, figure (b). Nomenclature: $\mathbf{F}=$ flammable mixture; $(\mathrm{NF})=$ Non-flammable mixture. Parameter values: heat-transfer parameter, $J=1$; inflow pressure, $\mathscr{P}^{*}=6.7$; inflow temperature, $T_{0}^{*}=1.5$.

there is no solution branch on the isola representing a stable combustion state.

Figure 5 (a) shows the $14^{2}$ steady-state diagram when the inert fraction is zero. (Note that the $\mathrm{H} 2$ and $\mathrm{H} 3$ Hopf points are too close to be separated visually.) There are now four supercritical Hopf points on the isola $(\alpha=0.1,0.1755,0.17734$ and 0.6324$)$. The region on the isola between the $(\mathrm{H} 2)$ and $(\mathrm{H} 3)$ points is unstable. Defining a mixture to be flammable when the system corresponds to a 'stable flame' then the I4 steady-state structure has two regions of flammability: $0.1<\alpha<0.1755$ and $0.17734<\alpha<0.6324$.

In Figure 5 (b) the Hopf bifurcation points are unfolded with the inert fraction. As the inert fraction increases, the $(\mathrm{H} 1)$ and $(\mathrm{H} 2)$ points approach each other whilst the (H3) and (H4) points approach each other. For the fuel fraction slightly greater than 0.2 there is a double Hopf bifurcation point and the $(\mathrm{H} 1)$ and $(\mathrm{H} 2)$ points disappear from the steady-state diagram. As the inert fraction is increased further the remaining Hopf bifurcation points become closer. A second double Hopf bifurcation occurs for a value of the inert fraction slightly below 0.35 . Thus as the inert fraction is increased through this value the fuel-air-inert mixture becomes non-flammable for any permitted value of the fuel fraction.

As explained in Subsection 3.1.2 the behaviour of a fuel-air-inert system in the adiabatic reactor can be predicted from the fuel-air bifurcation diagram. This remains true for the diabatic reactor. The sequence of bifurcation diagrams that is obtained as

\footnotetext{
${ }^{2}$ The I shows that the steady-state structure is an isola. The ' 4 ' denotes that there are four Hopf bifurcation points on the isola.
} 
the inert fraction is increased from zero can be predicted from the fuel-air bifurcation diagrams shown in [13]: at fixed inflow, temperature moves vertically down the bifurcation diagram. This is the same procedure used for the adiabatic reactor.

\section{Discussion}

\subsection{Change in flammability limits as the inert fraction is increased Flammability} limits are defined by the gain/loss of stability on the isola's combustion branch. In an adiabatic reactor these limits are identified with the extinction limit points of Figure 1. Inspection of the unfolding diagram, Figure 2 (b), reveals that as the inert fraction increases, the values of the fuel fraction at the points ELP 1 and ELP 2 increase and decrease respectively. Intuitively this is expected: increasing the value of the fuel fraction should make a marginally flammable mixture marginally non-flammable.

This intuitive behaviour is also exhibited in the diabatic reactor by the I2a unfolding diagram, Figure 4 (b). The values of the lower flammability limit (H1) and the upper flammability limit $(\mathrm{H} 2)$ increase and decrease respectively as the inert fraction increases. Thus marginally flammable mixtures become marginally non-flammable.

However, this intuitive behaviour is not exhibited by the (H3) bifurcation point in Figure 5 (b). In the absence of an inert $(\gamma=0)$ the value of the fuel fraction at the $\mathrm{H} 3$ point is given by $\alpha=0.196$. This value initially decreases as the inert fraction increases, an extrema of $\alpha=0.143$ being reached when $\gamma=0.287$. Thus, in this case, the addition of an inert species may make a marginally non-flammable mixture marginally flammable. This is counter-intuitive. (This figure also shows that the unfolding of the $(\mathrm{H} 1)$ and $(\mathrm{H} 2)$ bifurcation points is the expected 'intuitive' behaviour.)

4.2. Previous investigations The possibility of using the CSTR as a modelling tool to investigate flame inhibitors has received scant attention in the literature.

In an early study Brown and Schefer [3] modelled the effect that the addition of three additives, two physical and one chemical, had on the combustion of hydrogenoxygen-argon mixtures. They investigated how the addition of the additive changed the minimum residence time at which stable combustion occurs because "blowout parameters are specially sensitive to inhibitor type and concentration". This was done by solving the steady-state equations of their model (stability was not determined).

Babushok et al. [2] used the flow rate as the primary bifurcation parameter. Steadystate diagrams were determined for the combustion of methane in the presence and absence of physical and chemical inhibitors using detailed chemical mechanisms. The importance of this work is that by comparing the relative changes in the steadystate diagram, when an additive is added, against the known effectiveness of the additives the authors concluded that a ranking of flame suppression capability can 
be obtained from CSTR studies by examining conditions either near to extinction or near self-ignition. By the former it is meant the extinction limit point on a mushroom steady-state structure at high flow rates. This is a high-temperature process. The latter is the inflow temperature at which, at constant pressure, the isola singularity occurs, marking the transition from an isola steady-state diagram to a mushroom steady-state diagram. This is a low-temperature process.

A drawback of this study, as in the earlier work of Brown and Schefer [3], is that the stability of steady-state solutions was not determined. Thus these authors may have been investigating systems where, for example, the extinction mechanism was associated with a Hopf bifurcation point rather than at a limit point.

We can apply our approach to the two indicators of additive effectiveness identified by Babushok et al. The ability of flame inhibitors to induce high-temperature extinction would be identified by unfolding the bifurcation point at which extinction occurs (which we have shown is either a limit point or a Hopf bifurcation) with the additive fraction. A ranking of flame suppression capability then follows from comparing the additive fraction required to achieve a specified reduction in the value of the bifurcation point. A ranking of the additives in terms of their ability to prevent self-ignition would be determined by unfolding the isola singularity with the additive fraction.

This discussion suggests that it would be useful to apply our methodology to a problem using more detailed chemical mechanisms, interpreting suppressing capability using the indicators identified by Babushok et al. [2], as outlined in the preceding paragraph.

4.3. Heat-sink additives A second class of additives that can be incorporated into the current model is heat sinks. These are chemical species that take energy out of the flame through processes such as dissociation, decomposition and vaporisation. We present a brief investigation of this type of additive. This type of additive has previously been investigated from the perspective of thermal explosions in a batch reactor [11] and within the context of the propagation of a premixed laminar flame $[8,15]$. In addition to the aforementioned studies Ewing and co-workers [6] have developed a theory for heat-sink additives that predicts their ability to suppress a flame without requiring kinetic information.

To investigate a heat-sink additive, (2.4) and (2.5) are replaced by

$$
\begin{aligned}
\frac{\mathrm{d} \mathscr{I}^{*}}{\mathrm{~d} t^{*}}= & q_{2}^{*} \gamma-\left(q_{1}^{*}+q_{2}^{*}\right) \mathscr{I}^{*}-A_{a}^{*} e^{-E_{a}^{*} / T^{*} \mathscr{I}^{*}} \text { and } \\
\frac{\mathrm{d} T^{*}}{\mathrm{~d} t^{*}}= & \left(q_{1}^{*}+q_{2}^{*}\right)\left(T_{0}^{*}-T^{*}\right)+\frac{Q_{2}^{*} A_{2}^{*} \mathscr{P}^{*^{2}}}{T_{0}^{*^{2}}} e^{-E_{i}^{*} / T^{*} \mathscr{B}^{*} \mathscr{O}_{2}^{*}} \\
& -\frac{Q_{a}^{*} A_{a}^{*} \mathscr{P}^{*}}{T_{0}^{*}} e^{-E_{a}^{*} / T^{*}}-J\left(T^{*}-T_{a}^{*}\right)
\end{aligned}
$$




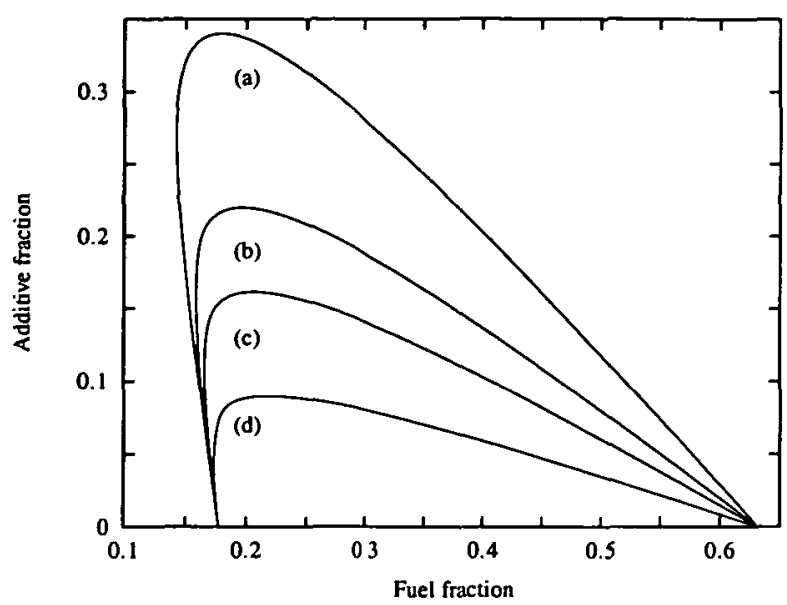

FIGURE 6. Unfolding of the (H3) and (H4) bifurcation points in the $\mathrm{I} 4$ steady-state diagram shown in Figure 5 (a) for a heat-sink additive. Parameter values: heat-transfer parameter, $J=1$; inflow pressure, $\mathscr{P}^{*}=6.7$; inflow temperature, $T_{0}^{*}=1.5$. Heat-sink parameter values: (a) $Q_{a}^{*}=0$, (b) $Q_{a}^{*}=0.1 Q_{2}^{*}$, (c) $Q_{a}^{*}=0.2 Q_{2}^{*}$, (d) $Q_{a}^{*}=0.5 Q_{2}^{*} ; A_{a}^{*}=A_{2}^{*} ; E_{a}^{*}=E_{2}^{*}$.

respectively.

Figure 6 shows an unfolding of the (H3) and (H4) Hopf bifurcation points in the I4 steady-state diagram of Figure 5 (b). (For clarity the unfolding of (H1) and (H2) are not shown.) Line (a) corresponds to the case $Q_{a}^{*}=0$, in which case the additive acts purely as an inert species; this line is identical to the $(\mathrm{H} 3 / \mathrm{H} 4)$ unfolding of Figure 5 (b). Line (a) bounds the behaviour of any heat-sink additive with $Q_{a}^{*}>0$. As the endothermicity of the heat-sink reaction increases, the amount of additive required to render a mixture containing a specified fuel fraction non-flammable decreases. Additionally the critical value of the additive fraction, at which all fuel-oxygen-inert mixtures become non-flammable, decreases as the endothermicity of the heat sink reaction increases. This value is the inert fraction at the double Hopf point at which the (H3) and (H4) points are annihilated. One could investigate the performance of a heat-sink additive in more detail by examining how, for fixed endothermicity, this critical value depends upon the kinetic parameters $A_{a}^{*}$ and $E_{a}^{*}$. Finally, Figure 6 shows that the non-intuitive decreasing of the lower flammability limit is present when an inert additive is replaced by a heat-sink additive, becoming more pronounced as the degree of endothermicity increases.

4.4. Limitations of the present study In this study we have assumed that the physical properties of the reaction mixture are independent of its composition. This is a convenient simplifying assumption from which it follows that all chemically inert species are equally effective in reducing flammability. In practice the efficiency of 
inerts is proportional to their heat capacity [7]. It is straightforward to include the heat capacity of the additive into our model. We have not done this here since, given the use of such a simple thermokinetic mechanism, we feel it is more constructive to outline our method rather than to try and draw detailed conclusions about the effectiveness of any particular additive.

4.4.i. The reaction rate $k_{1}$ We have assumed that the inert species has no chemical effect, that is, it does not change the value of the reaction rates $k_{1}$ and $k_{2}$. A referee has pointed out that if reaction (1.1) is taken literally as written then it is "first order and probably unimolecular". Assuming that this process can be described by the Lindemann-Hinshelwood mechanism then the value of $k_{1}$ would depend upon the concentration of the inert in a "well-known" way [1, Chapter 28.3] [10, Chapters $1-3]$.

A key assumption here is that reaction (1.1) can be "taken literally as written", so that the decay of the precursor species is governed by the Lindemann-Hinshelwood mechanism. The reaction-scheme given by (1.1) and (1.2) is based on the Sal'nikov scheme that was introduced to model the phenomena of cool-flame oscillations. It is very unlikely that this reaction represents a simple unimolecular reaction.

Assuming that the reaction is a simple unimolecular reaction then it should be noted that Figures 2 (b), 4 (b), 5 (b) and 6 represent experiments at constant pressure. Assuming that the inert is equally effective as oxygen in creating/de-energising the energised molecule appearing in the Lindemann-Hinshelwood mechanism it follows that there is no change in the overall reaction rate $\left(k_{1}\right)$ as the inert replaces oxygen.

The only results in this paper where the dependence of the Lindemann-Hinshelwood reaction rate upon the concentration of inert might be important are those presented in Figure 3 (a). However, the dependence of a unimolecular reaction rate upon the pressure of an inert species reaches a limiting asymptote at sufficiently high inert pressures. For a suitable choice of parameters in the Lindemann-Hinshelwood mechanism it can be arranged that the important pressure values in this figure are 'sufficiently high'.

Thus a strong case can be made that there is no need to take into account the effects of the inert species on reaction rate $k_{1}$.

4.4.2. The reaction rate $k_{2}$ A referee has suggested that "the second order reaction (1.2) would probably be affected by the inert acting as a third body and increasing the order of the reaction". However, it is well known in the combustion community that the retardancy effect of physical fire retardants is due to the combined effect of diluting the reaction mixture with the change in heat capacity of the reaction mixture $[5,7,9]$. In fact it is possible to accurately calculate flammability limits of fuel/diluent mixtures without any knowledge of the flame chemistry [16].

It should be recognised that reaction (1.2) is a global mechanism. The reaction 
scheme that gives this rate law is not a simple single-step bimolecular collision. It is therefore incorrect to model the effect of any additive by assuming that reaction (1.2) represents a single-step bimolecular collision.

Thus we can conclude that the second-order reaction (1.2) is not affected by the inert.

\section{Conclusion}

We have shown how the change in flammability of a fuel/air mixture when an inert additive is added can be investigated by unfolding the defining bifurcation points with the fraction of additive. We have also outlined how heat-sink additives can be incorporated into our model. For the systems considered, the ultimate quenching of a stable steady flame is associated with either an isola singularity, in an adiabatic reactor, or a double Hopf bifurcation, in a diabatic reactor.

Intuitively one expects that increasing the concentration of inert in fuel-air-inert mixtures kept at constant pressure would increase the lower flammability limit whilst decreasing the upper limit flammability limit. We have shown that this scenario can occur. However, the unfolding of the (H3) Hopf bifurcation point in Figures 5 (b) and 6 shows that a non-intuitive situation can arise whereby increasing the additive concentration can render a marginally non-flammable mixture flammable.

Although we considered the simplest possible mode of action for an additive with a very simple chemical mechanism our methodology can be applied to more realistic oxidation mechanisms coupled to more detailed additive chemistry. Future work is suggested combining this approach with the results established by Babushok $e t$ al. [2] regarding the specific bifurcations/degeneracies that can be used to rank flame suppression capability at low and high temperatures.

\section{Acknowledgements}

During this work I was supported by a grant from the Australia Research Council to work at UNSW@ADFA. I acknowledge the support and encouragement of Dr H. Sidhu during this work. Thought-provoking comments by a referee are gratefully acknowledged.

\section{Appendix A. Nomenclature}

In the following we note what the dimensionless variables mean. We refer to our earlier paper [12] for their definitions in terms of dimensional variables. However, we include both the dimensional and dimensionless variables associated with properties of the additive. Definitions of dimensional variables not defined here are in [12]. 
$A_{1}^{*} \quad$ Dimensionless rate-constant for the decomposition of the precursor species.

$A_{2}^{*} \quad$ Dimensionless pre-exponential factor for the oxidation reaction.

$A_{a} \quad$ Pre-exponential factor for decomposition of the heat-sink additive. $\left(\mathrm{s}^{-1}\right)$

$A_{a}^{*} \quad$ Dimensionless pre-exponential factor for decomposition of the heat-sink additive $A_{a}^{*}=A_{a} c_{p_{s}} \rho_{g} V_{g} /(\chi S)$.

$\mathscr{B}^{*} \quad$ The dimensionless concentration of gaseous fuel $\mathscr{B}$ in the reactor.

$\mathscr{B}^{*}(0)$ The dimensionless concentration of species $\mathscr{B}$ in the reactor at time $t^{*}=0$.

$E_{2}^{*} \quad$ Dimensionless activation energy for the oxidation reaction.

$E_{a} \quad$ Activation energy for the heat-sink reaction. $\left(\mathrm{Jmol}^{-1}\right.$ )

$E_{a}^{*} \quad$ Dimensionless activation energy for the heat-sink reaction $E_{a}^{*}=E_{a} / R T_{r}$.

$\mathscr{F}^{*} \quad$ The dimensionless concentration of the precursor species in the reactor.

$\mathscr{F}_{0}^{*} \quad$ The dimensionless concentration of the precursor species in the reactant inflow tube.

$\mathscr{I}$ The concentration of the inert additive in the reactor. $\left(\mathrm{mol} \mathrm{m}^{-3}\right)$

$\mathscr{I}^{*} \quad$ The dimensionless concentration of the inert additive in the reactor $\mathscr{I}^{*}=\mathscr{I} / c_{r}$.

$\mathscr{I}_{0} \quad$ The concentration of the inert additive in the inflow $\mathscr{I}_{0}=\mathscr{P}_{1} / R T_{0} \cdot\left(\mathrm{mol} \mathrm{m}^{-3}\right)$

$\mathscr{I}_{0}^{*} \quad$ The dimensionless concentration of the inert additive in the inflow $\mathscr{I}_{0}^{*}=\mathscr{I}_{0} / c_{r}=\mathscr{P}_{i} / \mathscr{P}_{0}=\gamma$.

$\mathscr{I}(0)$ The concentration of the inert additive in the reactor at time $t=0 .\left(\mathrm{mol} \mathrm{m}^{-3}\right)$

$\mathscr{I}^{*}(0)$ Dimensionless inert concentration in the reactor at time $t^{*}=0$ $\mathscr{I}^{*}(0)=\mathscr{I}(0) / c_{r}$.

$J$ A constant. $J=0$ and 1 correspond to adiabatic and diabatic operations respectively.

$\mathscr{O}_{2}^{*} \quad$ The dimensionless concentration of oxygen in the reactor.

$\mathscr{O}_{2}^{*}(0)$ The dimensionless oxygen concentration in the reactor at time $t=0$.

$\mathscr{P}^{*} \quad$ Dimensionless total pressure.

$\mathscr{P}_{f}$ The pressure of the precursor species flowing into the reactor. $\left(\mathrm{Jm}^{-3}\right)$.

$\mathscr{P}_{i} \quad$ The pressure of the inert species flowing into the reactor. $\left(\mathrm{Jm}^{-3}\right)$

$\mathscr{P}_{0} \quad$ The total pressure of the inflow reactants $\mathscr{P}_{0}=\mathscr{P}_{\text {air }}+\mathscr{P}_{f}+\mathscr{P}_{i} \cdot\left(\mathrm{Jm}^{-3}\right)$

$Q_{2}^{*} \quad$ Dimensionless exothermicity of the oxidation reaction.

$Q_{a} \quad$ Endothermicity of the heat-sink reaction. $\left(\mathrm{Jmol}^{-1}\right)$

$Q_{a}^{*} \quad$ Dimensionless endothermicity of the heat-sink reaction

$Q_{a}^{*}=Q_{a}\left(\mathscr{P}_{r} / R T_{r}\right)\left(1 / c_{p_{s}} \rho_{g} T_{r}\right)$.

$T^{*} \quad$ The dimensionless temperature inside the reactor.

$T^{*}(0)$ The dimensionless temperature inside the reactor at time $t^{*}=0 .(\mathrm{K})$

$T_{a}^{*} \quad$ The dimensionless temperature of the reactor walls.

$T_{0} \quad$ The temperature of the inflow. (K)

$T_{0}^{*} \quad$ The dimensionless temperature of the inflow. 
$q_{1}^{*} \quad$ The dimensionless flow rate through the reactant tube into the reactor.

$q_{2}^{*} \quad$ The dimensionless flow rate through the air tube into the reactor.

$t^{*} \quad$ Dimensionless time.

$\alpha \quad$ The fraction of the total inflow concentration comprised of the pre-cursor species $\alpha=\mathscr{P}_{f} / \mathscr{P}_{0}$.

$\gamma \quad$ The fraction of the total inflow concentration comprised of the inert species $\gamma=\mathscr{P}_{i} / \mathscr{P}_{0}$.

Parameter values: $A_{1}^{*}=0.1, A_{2}^{*}=0.661 \times 10^{9}, E_{2}^{*}=46.308, Q_{2}^{*}=2828.074$, $q_{1}^{*}=q_{2}^{*}=0.00101$.

\section{References}

[1] P. W. Atkins, Physical Chemistry, 3rd ed. (Oxford Univ. Press, Oxford, 1987).

[2] V. Babushok, T. Noto, D. R. F. Burgess, A. Hammins and W. Tsang, "Inhibitor influence on the bistability of a CSTR", Combustion and Flame 108 (1997) 61-70.

[3] N. J. Brown and R. W. Schefer, "A computational study of physical and chemical inhibition in a perfectly stirred reactor", Fire and Materials 5 (1981) 14-23.

[4] E. J. Doedel, T. F. Fairgrieve, B. Sandstede, A. R. Champneys, Y. A. Kuznetsov and X. Wang, "AUTO 97: Continuation and bifurcation software for ordinary differential equations (with HomCont)", March 1998. Available by anonymous ftp from ftp.cs.concordia.ca/pub/doedel/auto.

[5] D. Drysdale, An introduction to fire dynamics, 2nd ed. (John Wiley and Sons, New York, 1999).

[6] C. T. Ewing, C. L. Beyler and H. W. Carthard, "Extinguishment of class B flames by thermal mechanisms: Principles underlying a comprehensive theory; prediction of flame extinguishing effectiveness", J. Fire Protection Engng 6 (1994) 23-54.

[7] I. Glassman, "Flammability limits", in Combustion, 3rd ed., Chapter 4D.1, (Academic Press, New York, 1996) 162-170.

[8] B. F. Gray, S. Kalliadasis, A. Lazarovich, C. Macaskill, J. H. Merkin and S. K. Scott, "The suppression of an exothermic branched-chain flame through endothermic reaction and radical scavenging", Proc. Roy. Soc. London A 458 (2002) 2119-2138.

[9] J. F. Griffiths and J. A. Barnard, Flame and Combustion, 3rd ed. (Blackie Academic and Professional, London, 1995).

[10] M. F. R. Mulcahy, Gas kinetics, Studies in Modern Chemistry (Nelson, London, 1973).

[11] M. I. Nelson, "Decomposition and inhibition schemes for polymeric combustion", Ph. D. Thesis, Dept of Appl. Math. Studies, The University of Leeds (UK), 1994.

[12] M. I. Nelson and H. S. Sidhu, "Bifurcation phenomena for an oxidation reaction in a continuously stirred tank reactor. I Adiabatic operation", J. Math. Chem. 31 (2002) 155-186.

[13] M. I. Nelson and H. S. Sidhu, "Bifurcation phenomena for an oxidation reaction in a continuously sturred tank reactor. II Diabatic operation", The ANZIAM J. 45 (2004) 303-326.

[14] H. S. Sidhu, M. I. Nelson, G. N. Mercer and R. O. Weber, "Dynamical analysis of an elementary $X+Y \rightarrow P$ reaction in a continuously stirred tank reactor", J. Math. Chem. 28 (2000) 353-375.

[15] P. L. Simon, S. Kalliadasis, J. H. Merkin and S. K. Scott, "Quenching of flame propagation through endothermic reaction", J. Math. Chem. 32 (2002) 73-98.

[16] I. Wierzba, S. O. Bade, O. B. Shrestha and G. A. Karim, "An approach for predicting the flammability limits of fuel/diluent mixtures in air", J. Institute of Energy 69 (1996) 122-130. 